\title{
A MODULAR INHERENTLY NONFINITELY BASED LATTICE
}

\author{
RALPH FREESE, GEORGE MCNULTY, AND J. B. NATION
}

In [4] we gave a construction of inherently nonfinitely based lattices which produced a wide variety of examples. But none of these examples was modular and we asked in Problem 1 for a modular example. Here we shall show that $\mathbf{L}_{\infty}$ of Figure 1 is such an example.

Theorem 1. $\mathbf{L}_{\infty}$ is an inherently nonfinitely based modular lattice.

Proof. As observed in McNulty [6], a locally finite variety $\mathcal{V}$ of finite type is inherently nonfinitely based if and only if for infinitely many natural numbers $N$, there is a non-locally-finite algebra each of whose $N$-generated subalgebras belongs to $\mathcal{V}$. We prove the theorem by establishing these facts. We assume the reader is familiar with the basic facts of modular lattices; see $[1],[2],[5]$.

Let $\mathbf{B}$ (for bottom) be the sublattice of $\mathbf{L}_{\infty}$ consisting of all elements of finite height and let $\mathbf{T}$ consist of all elements of finite depth. Of course $\mathbf{L}_{\infty}$ is the ordinal (or linear) sum $\mathbf{B}+\mathbf{T}$ of these sublattices.

Lemma 2. The variety $\mathbb{V}\left(\mathbf{L}_{\infty}\right)$ generated by $\mathbf{L}_{\infty}$ is locally finite.

Proof. To prove this we need to show that for every finite $n$ there is a bound on the size of the $n$-generated subalgebras of $\mathbf{L}_{\infty}$. We do this by induction on $n$. Suppose that $x_{1}, \ldots, x_{n}$ are elements of $\mathbf{L}_{\infty}$ and let $\mathbf{S}$ be the sublattice they generate. We may assume that all of these elements either lie in $\mathbf{B}$ or they all lie in $\mathbf{T}$ since otherwise $\mathbf{S}$ is the ordinal sum of two sublattices with fewer generators. By duality we may assume they all lie in B. Thus each $x_{k}$ has a rank (or height) $r_{k}$.

Observe that if $a$ and $b$ are elements of $\mathbf{B}$ with ranks $r_{a}$ and $r_{b}$ and $r_{b}-r_{a} \geq 4$ then the meet of all elements with rank at least $r_{b}$ is greater than or equal to the join of all elements with rank at most $r_{a}$. So if we let $r_{k}$ be the rank of $x_{k}$ and (re)order the $x_{k}$ 's so that $r_{1} \leq r_{2} \leq \cdots \leq r_{n}$ then we may assume $r_{k+1}-r_{k} \leq 3$ since otherwise $\mathbf{S}$ is a ordinal sum of sublattices with fewer generators. Thus $\mathbf{S}$ lies in an interval of $\mathbf{L}_{\infty}$ which has length at most $3(n+2)$. All intervals of $\mathbf{L}_{\infty}$ of fixed length have a bound on their size. Thus $\mathbf{S}$ is of bounded size.

Let $\mathbf{M}_{3}$ be the five element modular, nondistributive lattice and let $\mathbf{Z}$ be integers as a chain. Let $\mathbf{M}_{3}[\mathbf{Z}]$ be the lattice of all order-preserving functions

Date: June 1, 2004. 

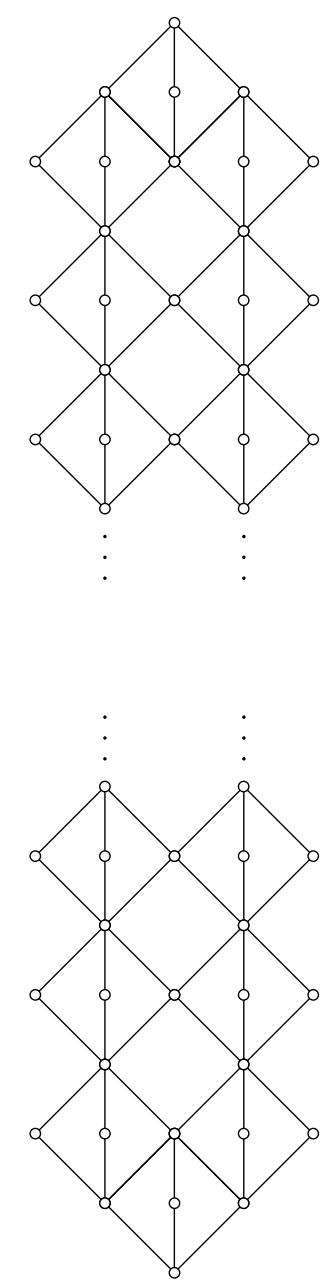

FIGURE 1. $\mathbf{L}_{\infty}$, an inherently nonfinitely based modular lattice

from $\mathbf{Z}$ to $\mathbf{M}_{3}$. If $x \in \mathbf{M}_{3}$ let $\bar{x}$ denote the constant map. The following facts are easily established:

(1) $\mathbf{M}_{3}[\mathbf{Z}]$ is a subdirect power of $\mathbf{M}_{3}$ and so in the variety generated by $\mathbf{M}_{3}$.

(2) $x \mapsto \bar{x}$ embedds $\mathbf{M}_{3}$ in $\mathbf{M}_{3}[\mathbf{Z}]$.

(3) If $v \prec u$ in $\mathbf{M}_{3}$, then the interval $[\bar{v}, \bar{u}]$ is $\mathbf{1}+\mathbf{Z}+\mathbf{1}$ (the ordinal sum).

(4) If $a$ is an atom of $\mathbf{M}_{3}$ and 0 is its least element, then $\mathbf{M}_{3}[\mathbf{Z}]$ is generated by the constanst maps and the interval $[\overline{0}, \bar{a}]$.

(5) The (left) shift operator $\sigma[(\sigma f)(i)=f(i+1)]$ is an automorphism of $\mathbf{M}_{3}[\mathbf{Z}]$.

We wish do something similar with $\mathbf{L}_{\infty}$ and other modular lattices. So let $\mathbf{L}$ be a modular lattice. We start by forming $\mathbf{M}$ the lattice of all orderpreserving maps from $\mathbf{Z}$ into $\mathbf{L}$. This lattice is bigger than we want; we 
would like that intervals of $\mathbf{L}$ which are chains remain chains in $\mathbf{M}$ under the natural (diagonal) embedding (denoted $x \mapsto \bar{x}$ ) of $\mathbf{L}$ into $\mathbf{M}$. To do this we take the sublattice of $\mathbf{M}$ whose universe is

$$
\{x \in \mathbf{M}: \bar{v} \leq x \leq \bar{u} \text {, for some } u, v \in \mathbf{L} \text { with }[v, u] \text { complemented in } \mathbf{L}\}
$$

The following lemma proves that this is (the universe of) a sublattice of $\mathbf{M}$. We donote this lattice by $\mathbf{L}[\mathbf{Z}]$.

Lemma 3. If $\left[a_{0}, a_{1}\right]$ and $\left[b_{0}, b_{1}\right]$ are complemented intervals in a modular lattice $\mathbf{L}$, then $\left[a_{0} \vee b_{0}, a_{1} \vee b_{1}\right]$ is also complemented.

Proof. Let $c_{0}=a_{0} \vee b_{0}$ and $c_{1}=a_{1} \vee b_{1}$. Let $x \in\left[c_{0}, c_{1}\right]$. Let $d=a_{1} \vee b_{0}$ and $e=a_{0} \vee b_{1}$. Since $\left[c_{0}, d\right]$ is a transpose of a subinterval of $\left[a_{0}, a_{1}\right]$ it is complemented and similarly $\left[c_{0}, e\right]$ is complemented. Let $y$ be a complement of $x \wedge d$ in $\left[c_{0}, d\right]$ and let Let $z$ be a complement of $(x \vee d) \wedge e$ in $\left[c_{0}, e\right]$. Then $y \vee z$ is a complement of $x$ in $\left[c_{0}, c_{1}\right]$. Indeed

$$
\begin{aligned}
x \wedge(y \vee z) & =x \wedge(x \vee d) \wedge(y \vee z)=x \wedge[y \vee((x \vee d) \wedge z)] \\
& =x \wedge[y \vee((x \vee d) \wedge e \wedge z)]=x \wedge\left[y \vee c_{0}\right] \\
& =x \wedge y=x \wedge d \wedge y=c_{0} \\
x \vee y \vee z & =x \vee(x \wedge d) \vee y \vee z \\
& =x \vee d \vee z=x \vee d \vee e=c_{1}
\end{aligned}
$$

Now we turn to constructing non-locally-finite lattices whose $N$-generated sublattices lie in $\mathbb{V}\left(\mathbf{L}_{\infty}\right)$. For $n$ an even integer at least 4 , B has 5 elements of height $n$, only one of which is join reducible (the middle one). We let $\mathbf{K}_{n}$ denote this ideal. It has two coatoms and we let $\mathbf{L}_{n}$ be the lattice obtained from $\mathbf{K}_{n}$ by adding another coatom which is above the meet of these two coatoms. $\mathbf{L}_{10}$ is diagrammed in Figure 2.

We shall modify $\mathbf{L}_{n}[\mathbf{Z}]$ into a lattice $\mathbf{L}_{n}[\mathbf{Z}]^{*}$ using the Hall-Dilworth gluing construction which we now review.

If a lattice $\mathbf{L}$ has a filter which is isomorphic to the ideal of another lattice $\mathbf{K}$ then we can identify each element of the filter with corresponding element of the ideal and order the elements of $L \cup K$ (with these identifications) by the transitive closure of the orders on $\mathbf{L}$ and $\mathbf{K}$. The result is a lattice $\mathbf{M}$ and, if both $\mathbf{L}$ and $\mathbf{K}$ are modular, the $\mathbf{M}$ is also. This is the famous HallDilworth gluing construction. [add some refs.] $\mathbf{L}$ is an ideal of $\mathbf{M}$ and $\mathbf{K}$ is a filter and $L \cup K=M$. Conversely if a lattice $\mathbf{M}$ is the union of an ideal $\mathbf{L}$ and a filter $\mathbf{K}$ and $L \cup K=M$ and $L \cap K \neq \emptyset$ then $\mathbf{M}$ is the Hall-Dilworth gluing of $\mathbf{L}$ and $\mathbf{K}$ over their intersection using the identity map.

If both $\mathbf{L}$ and $\mathbf{K}$ are copies of $\mathbf{M}_{3}$ and $a$ is an atom of $\mathbf{L}$ and $b$ is an atom of $\mathbf{K}$ then we can apply this construction using the filter of $\mathbf{L}$ generated by $a$ and the ideal of $\mathbf{K}$ generated by $b$ to obtain the lattice $\mathbf{M}_{33}$ of Figure 3 .

Similarly $\mathbf{M}_{33}[\mathbf{Z}]$ is the Hall-Dilworth gluing of two copies of $\mathbf{M}_{3}[\mathbf{Z}]$ over the interval from $\bar{a}$ to $\bar{b}$. To prove this using the remarks above one only 


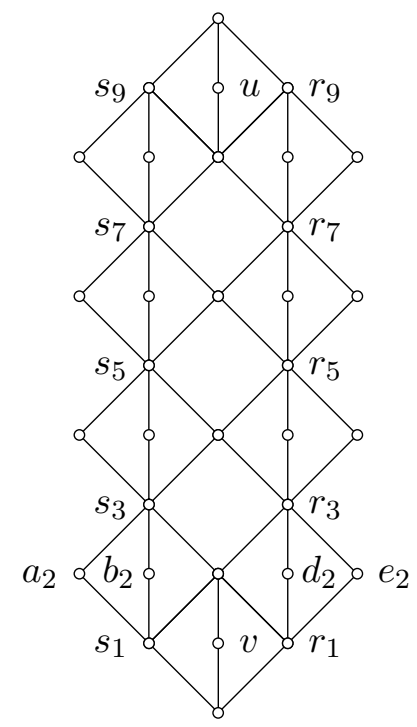

Figure 2. $\mathbf{L}_{10}$

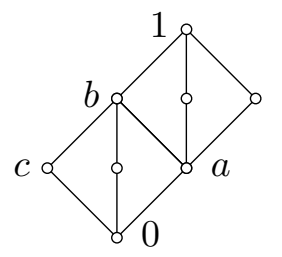

Figure $3 . \mathbf{M}_{33}$

needs to verify that every element of $\mathbf{M}_{33}[\mathbf{Z}]$ is either below $\bar{b}$ or above $\bar{a}$, which is straightforward.

A typical element of $[\bar{a}, \bar{b}]$ is $c_{i}$, where $c_{i}$ is the function $c_{i}(k)=a$ if $k \leq i$ and $b$ otherwise. Instead of using the identity map when gluing the two

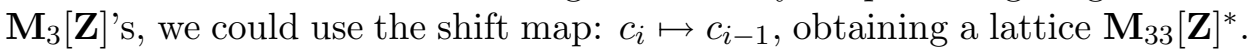
It is easy to check that $\mathbf{M}_{33}[\mathbf{Z}]^{*} \cong \mathbf{M}_{33}[\mathbf{Z}]$.

In $\mathbf{L}_{n}-\{u, v\}$ there are two element of each odd dimension. We can arrange these elements into two chains $s_{1}<s_{3}<\cdots<s_{n-1}$ and $r_{1}<r_{3}<$ $\cdots<r_{n-1}$; see Figure 2 .

We have $s_{1}<r_{3}$ and one can verify that every element in $\mathbf{L}_{n}[\mathbf{Z}]$ is either in the principal ideal $\mathbf{I}$ generated by $\bar{r}_{3}$ or in the principal filter $\mathbf{F}$ generated by $\bar{s}_{1}$ and thus $\mathbf{L}_{n}[\mathbf{Z}]$ is the Hall-Dilworth gluing of $\mathbf{I}$ and $\mathbf{F}$. $\mathbf{I}$ is isomorphic to $\mathbf{M}_{33}[\mathbf{Z}]$. Let $\mathbf{L}_{n}[\mathbf{Z}]^{*}$ be the result of gluing $\mathbf{M}_{33}[\mathbf{Z}]^{*}$ to $\mathbf{F}$ using the identity map on the interval $\left[\bar{s}_{1}, \bar{r}_{3}\right]$. 
Unlike the situation with $\mathbf{M}_{33}[\mathbf{Z}]^{*}, \mathbf{L}_{n}[\mathbf{Z}]^{*}$ is not isomorphic to $\mathbf{L}_{n}[\mathbf{Z}]$. In fact $\mathbf{L}_{n}$ has the sequence of transpositions

$$
\begin{aligned}
{[0, v] \nearrow } & {\left[a_{2}, s_{3}\right] \searrow\left[s_{1}, b_{2}\right] \nearrow\left[e_{4}, r_{5}\right] \searrow\left[r_{3}, d_{4}\right] } \\
& \cdots \nearrow[u, 1] \searrow \cdots \searrow\left[r_{1}, d_{2}\right] \nearrow\left[e_{2}, r_{3}\right] \searrow[0, v]
\end{aligned}
$$

where $a_{i}, b_{i} \in\left[s_{i-1}, s_{i+1}\right]$ and $d_{i}, e_{i} \in\left[r_{i-1}, r_{i+1}\right]$ are the four irreducible elements of rank $i, i$ even.

Of course this defines an automorphism on $[\overline{0}, \bar{v}]$ in $\mathbf{L}_{n}[\mathbf{Z}]$ but since the sequence of transpositions goes through the shifted interval, this automorphism is the shift, sending each element $x$ with $\overline{0}<x<\bar{v}$ to its lower cover. Thus the sublattice of $\mathbf{L}_{n}[\mathbf{Z}]^{*}$ generate by $\mathbf{L}_{n}$ and any element $\overline{0}<x<\bar{v}$ is infinite (in fact it is all of $\mathbf{L}_{n}[\mathbf{Z}]^{*}$, but we do not use this fact).

In order to complete the proof of Theorem 1 it suffices to show that for each $N$ we can choose $n$ large enough so that the $N$-generated sublattices of $\mathbf{L}_{n}[\mathbf{Z}]^{*}$ lie in $\mathbb{V}\left(\mathbf{L}_{\infty}\right)=\mathbb{V}\left(\mathbf{L}_{\infty}[\mathbf{Z}]\right)$. As usual we view $\mathbf{L}_{n}$ as embedded in $\mathbf{L}_{n}[\mathbf{Z}]^{*}$ by the diagonal map. Every element of $x \in \mathbf{L}_{n}[\mathbf{Z}]^{*}$ lies in a uniquely determined complemented interval $\left[z_{x}, t_{x}\right]$ of $\mathbf{L}_{n}$ of minimal dimension. $z_{x}$ is just the join of all elements of $\mathbf{L}_{n}$ below $x$ and $t_{x}$ is defined dually. Of course the dimension (in $\mathbf{L}_{n}$ ) of $\left[z_{x}, t_{x}\right]$ is at most 2 . Thus we may assign to each element $x$ of $\mathbf{L}_{n}[\mathbf{Z}]^{*}$ an lower and upper rank (the ranks in $\mathbf{L}_{n}$ of $z_{x}$ and $t_{x}$ ) and these differ by at most 2 . So if we let $\mathbf{S}$ be an $N$-generated sublattice of $\mathbf{L}_{n}[\mathbf{Z}]^{*}$ then an argument similar to the proof of Lemma 2 shows that, if $\mathbf{S}$ is linearly indecomposable, it lies in an interval $[\bar{a}, \bar{b}]$ of $\mathbf{L}_{n}[\mathbf{Z}]^{*}$ where the dimension of $[a, b]$ in $\mathbf{L}_{n}$ is at most $5(N+2)$. It follows that, for any $N$-generated sublattice $\mathbf{S}$, if $n>5(N+2)+4$ then either $\mathbf{S}$ lies in the filter $\left[\bar{r}_{1} \vee \bar{s}_{1}, \overline{1}\right]$ or the ideal $\left.\overline{0}, \bar{r}_{n-1} \wedge \bar{s}_{n-1}\right]$ or is the linear sum of two such lattices. So it suffices to show that this filter and ideal are isomorphic to sublattices of $\mathbf{L}_{\infty}[\mathbf{Z}]$. For the filter this is straightforward since the filter does not contain the shifted interval.

Let $\mathbf{I}$ denote the ideal. To see that it is also embedable into $\mathbf{L}_{\infty}[\mathbf{Z}]$ let $\mathbf{P}_{n}$ be the filter [ $\left.s_{1}, 1\right]$ of $\mathbf{K}_{n}$. (Recall $\mathbf{K}_{n}$ is $\mathbf{L}_{n}$ with $u$ removed.)

Now $\mathbf{I}$ is isomorphic it $\mathbf{K}_{n}[\mathbf{Z}]^{*}$ and $\mathbf{K}_{n}[\mathbf{Z}]$ is naturally embeddable into $\mathbf{B}$ and so into $\mathbf{L}_{\infty}[\mathbf{Z}]$. Thus it suffices to show that $\mathbf{K}_{n}[\mathbf{Z}] \cong \mathbf{K}_{n}[\mathbf{Z}]^{*}$. In $\mathbf{P}_{n}$ every prime interval is projective with either $\left[r_{1} \vee s_{1}, s_{3}\right]$ or $\left[r_{1} \vee s_{1}, r_{3}\right]$, but not both. So $\mathbf{P}_{n}$ is a subdirect product of two lattices, say $\mathbf{Q}$ and $\mathbf{R}$, and $\mathbf{P}_{n}[\mathbf{Z}]$ is a subdirect product of $\mathbf{Q}[\mathbf{Z}]$ and $\mathbf{R}[\mathbf{Z}]$. So $\mathbf{P}_{n}[\mathbf{Z}]$ has an automorphism $\tau$ which is the shift operator on one of these factors and the identity on the other. To make $\tau$ explicit let $x \in \mathbf{P}_{n}[\mathbf{Z}]$ and let $z_{x}$ and $t_{x}$ be the element of $\mathbf{P}_{n}$ defined above. If $z_{x}=t_{x}$ then $x \in \mathbf{P}_{n}$ and $\tau(x)=x$. Suppose the dimension of $\left[z_{x}, t_{x}\right]$ is 1 . If $\left[z_{x}, t_{x}\right]$ projects to $\left[r_{1} \vee s_{1}, r_{3}\right]$ then we apply the shift operator on $\left[\bar{z}_{x}, \bar{t}_{x}\right]$ to $x$; that is $\tau(x)$ is the unique upper cover of $x$ in $\left[\bar{z}_{x}, \bar{t}_{x}\right]$. If $\left[z_{x}, t_{x}\right]$ projects to $\left[r_{1} \vee s_{1}, r_{3}\right]$ then $\tau(x)=x$. If the dimension of $\left[z_{x}, t_{x}\right]$ is 2 then $\left[z_{x}, t_{x}\right]$ is isomorphic to either $\mathbf{M}_{3}$ or $\mathbf{2} \times \mathbf{2}$. In the former case if the prime quotients of $\left[z_{x}, t_{x}\right] \cong \mathbf{M}_{3}$ project to $\left[r_{1} \vee s_{1}, r_{3}\right]$ the we apply the shift operator to $x$, otherwise $x$ is fixed. Finally 
if $\left[z_{x}, t_{x}\right] \cong \mathbf{2} \times \mathbf{2}$ and $r$ and $s$ are the atoms of $\left[z_{x}, t_{x}\right]$, then $x=(x \wedge \bar{r}) \vee(x \wedge \bar{s})$ and $\tau(x)=\tau(x \wedge \bar{r}) \vee \tau(x \wedge \bar{s})$.

Now we define $\rho: \mathbf{K}_{n}[\mathbf{Z}] \rightarrow \mathbf{K}_{n}[\mathbf{Z}]^{*}$. If $x \geq \bar{s}_{1}$ we let $\rho(x)=\tau(x)$. If $x \nsucceq \bar{s}_{1}$ then $x \leq \bar{r}_{3}$. If such an $x$ is above $\bar{r}_{1}, \rho$ applies the shift operator to $x$; otherwise $\rho(x)=x$. Since $\left[0, \bar{r}_{3}\right]$ in $\mathbf{K}_{n}[\mathbf{Z}]^{*}$ is obtained from gluing two copies of $\mathbf{M}_{3}$ by applying the shift operator to the top copy, $\rho$ restricted to $\left[\overline{0}, \bar{r}_{3}\right]$ is an isomorphism. Finally one checks that $\rho$ is one-to-one and onto and that it preserves order and so is an isomorphism.

This completes the proof of Theorem 1.

\section{REFERENCES}

[1] P. Crawley and R. P. Dilworth, Algebraic theory of lattices, Prentice-Hall, Englewood Cliffs, New Jersey, 1973.

[2] B. A. Davey and H. A. Priestley, An introduction to lattices and order, Cambridge University Press, Cambridge, 1989.

[3] R. Freese, J. Ježek, and J. B. Nation, Lattices with large minimal extensions, Algebra Universalis 45 (2001), 221-309.

[4] Ralph Freese, George F. McNulty, and J. B. Nation, Inherently nonfinitely based lattices, Annals of Pure and Applied Logic 115 (2002), 175-193, online manuscript available at: http://www . math.hawaii.edu/ ralph/papers.html.

[5] Ralph McKenzie, George McNulty, and Walter Taylor, Algebras, lattices, varieties, Volume I, Wadsworth and Brooks/Cole, Monterey, California, 1987.

[6] G. McNulty, How to construct finite algebras which are not finitely based, Universal Algebra and Lattice Theory, Springer-Verlag, New York, 1985, S. Comer, ed., Lecture Notes in Mathematics, vol. 1149, pp. 167-174.

UNIVERSity OF HaWAiI, HoNOLUlu, HI 96822

E-mail address: ralph@math.hawaii.edu

University of South Carolina, Columbia, SC 29208

E-mail address: mcnulty@math.sc.edu

University of Hawait, Honolulu, HI 96822

E-mail address: jb@math.hawaii.edu 